\title{
Regulation of Intracellular and Extracellular Neutral and Alkaline Proteases in Aspergillus nidulans
}

\author{
By B. L. COHEN \\ Institute of Genetics, University of Glasgow, Glasgow, G I I ${ }_{5} J S$
}

(Received 29 May 1973)

\begin{abstract}
SUMMARY
Synthesis and secretion of extracellular neutral and alkaline proteases in Aspergillus nidulans are repressed in the presence of low molecular weight sources of carbon, nitrogen and sulphur; protease is formed and released when the medium is deficient for any one of these elements. Induction of protease by exogenous protein does not occur.

Activation of intracellular protease is repressed by sources of carbon, nitrogen, sulphur and phosphorus, activation taking place when any one is deficient.

These two systems of repression are independent of one another and of the two previously defined systems of ammonium repression in Aspergillus nidulans. Several classes of mutant selected for ammonium derepression also have abnormal carbon repression of protease.
\end{abstract}

\section{INTRODUCTION}

When growing on media containing a low molecular weight source of nitrogen, Aspergillus nidulans does not produce extracellular neutral and alkaline proteases and the intracellular protease $\beta$ is in an inactive or weakly active precursor form. When growing on media containing protein as sole nitrogen source, three extracellular proteases $(\alpha, \gamma-\delta$, and $\epsilon)$ are released into the medium and occur in the mycelium together with protease $\beta$ in its active form. This regulation of proteases by nitrogen metabolites has been called 'ammonium repression' (Cohen, 1972, I973 b; Pateman, Kinghorn, Dunn \& Forbes, 1973).

The experiments that established the occurrence of ammonium repression of proteases did not exclude the possibility of induction by exogenous protein. They did, however, indicate that if induction occurred it must have been subordinate to repression.

This paper describes experiments on wild-type and mutant strains of Aspergillus which demonstrate that protease formation is not induced but is regulated by carbon, nitrogen and sulphur repression; synthesis of protease takes place when any one of these is withheld. In addition it is shown that activation of the intracellular protease $\beta$ is regulated by carbon, nitrogen, sulphur and phosphorus repression.

\section{METHODS}

General techniques and strains. General techniques for Aspergillus nidulans were described by Pontecorvo et al. (I953). The strain biAI is a biotin-requiring derivative of the Glasgow wild-type and was Glasgow stock strain 05I (FGSC26). The mutant xprDI is insensitive to ammonium repression of protease and has been described previously (Cohen, I972); its other defects in ammonium regulation were described by Pateman et al. (I973). Recent results indicate that the $x$ pr $D_{I}$ recombinants in current use may carry an unidentified chromo- 
some aberration giving rise in crosses to a class of $x p r D^{+}$progeny unable to grow on complete medium. These were overlooked in the cross from which mutant recombinants were isolated because a full analysis was not made (Cohen, 1972). However, no results associate the aberration with the phenotypic effects of the $x p r D$ mutation.

The methylammonium-resistant mutants meaA8 and meaB6 were supplied by Dr H. N. Arst, University of Cambridge. Their defects in ammonium regulation and methylammonium transport and metabolism have been described by Arst \& Cove (1969), Arst \& Page (1973) and Pateman et al. (1973).

Glutamate dehydrogenase-deficient mutants $g d h A I$ (NADP-linked GDH) and $g d h B I$ (NAD-linked GDH) were supplied by Mr J. Kinghorn, University of Glasgow. The isolation, mapping and ammonium-regulation defects of these mutants have been described by Kinghorn \& Pateman (1973). DER-3 and $a m r A I$ were supplied by Professor J. A. Pateman, University of Glasgow; they are described by Pateman et al. (1973). Pateman \& Cove (1967) described nirAI and the Glasgow strain oI 28 (Cambridge I53) was used.

Media. Liquid minimal medium differed from that of Pontecorvo et al. (I953) principally in the substitution of ammonium for nitrate. It contained $50 \mathrm{~mm}$-glucose, $25 \mathrm{~mm}$-ammonium tartrate (replaced by $50 \mathrm{~mm}$-ammonium dihydrogen phosphate in $\mathrm{C}$-free medium and reduced to $5 \mathrm{~mm}$ - when testing $g d h A$ strains), II $\cdot 2 \mathrm{~mm}$-potassium dihydrogen phosphate, $7 \mathrm{~mm}$ potassium chloride, $2 \cdot 1 \mathrm{mM}$-magnesium sulphate and traces of ferrous and zinc sulphates. Minimal medium was supplemented with the nutrients required by the strains and all liquid growth and treatment media contained $0.001 \%(\mathrm{v} / \mathrm{v})$ Tween-80 wetting agent.

Growth in preparation for phosphorus-starvation was carried out in liquid minimal medium containing a growth-limiting concentration $(0.05 \mathrm{~mm})$ of potassium dihydrogen phosphate; effective phosphorus starvation could not be obtained in a transfer experiment if the phosphorus concentration during growth was higher.

Growth in preparation for cycloheximide treatment was carried out in medium containing Io mM-ammonium ion.

Treatment media for transfer experiments were obtained by modification of minimal medium, as indicated in Table I.

Growth conditions, transfer experiments, protease assay and electrophoresis. Exponentially growing mycelium was harvested from thin liquid layer cultures incubated at $37{ }^{\circ} \mathrm{C}$ for I 6 to $\mathrm{I} 8 \mathrm{~h}$ after inoculation with $\mathrm{Io}^{6}$ washed conidia/ml (Cohen, I973a). Mycelium was filtered on sterile gauze, washed with 100 vol. of treatment medium at $37^{\circ} \mathrm{C}$, resuspended in treatment medium at $37^{\circ} \mathrm{C}$, dispensed in $10 \mathrm{ml}$ portions into Petri dishes, and returned to the incubator. Such cultures contained I to $2 \mathrm{mg}$ dry wt of mycelium $/ \mathrm{ml}$ after treatment. At least two portions were pooled for each sample intended for determination of dry weight and extracellular protease specific activity; at least four portions were pooled for each sample intended for preparation of a mycelial extract. The methods of dry weight estimation, protease assay, specific activity calculation, mycelial extraction and protease electrophoresis on cellulose acetate have been described (Cohen, I973 $b$ ).

In preliminary experiments treatment was continued for 7 to $24 \mathrm{~h}$ but the results indicated that all changes in response to treatment could be observed during the first 5 to $7 \mathrm{~h}$.

In every transfer experiment involving nutrient limitation, cessation of growth was established by determinations of mycelial dry weight.

The $\mathrm{pH}$ of the medium in all experiments remained between 4.5 and 6.5 .

Plate tests for repression of proteases. The plate test for ammonium repression of proteases has been described (Cohen, 1972) and has been used here with minor modifications. Details of these and of media used in other plate tests for repression are contained in Table 2. 
Table I. Modified minimal media used in transfer experiments

Modified minimal medium*

Containing protein

Carbon-free

Nitrogen-free

Phosphorus-free

Sulphur-free
Substances omitted

Glucose

Ammonium tartrate

Ammonium tartrate

Potassium dihydrogen

phosphate

Magnesium sulphate
Substances added

(final concentrations)

$1 \%(w / v)$ Protein $\dagger$

$50 \mathrm{~mm}$-Ammonium dihydrogen phosphate

-

2.I mM-Magnesium chloride

* Unmodified minimal medium contained $50 \mathrm{~mm}$-glucose, $50 \mathrm{~mm}$-ammonium tartrate, I $\cdot 3 \mathrm{~mm}$-potassium dihydrogen phosphate and $2 \cdot 1$ mM-magnesium sulphate.

$\dagger$ Stock solution was $10 \%$ (w/v) skimmed milk (Oxoid Ltd. Southwark Bridge Rd., London S.E.I), dialysed overnight against running water and sterilized by autoclaving.

$\ddagger$ In some experiments $50 \mathrm{mM}$-tris- $\mathrm{HCl}(\mathrm{pH} 8)$ buffer was added. Little pH change occurred in its absence and no effects on protease regulation were noted.

\section{RESULTS}

Derepression of protease by nitrogen starvation and absence of induction by exogenous protein. When mycelium growing on minimal medium was transferred to nitrogen-free medium, intracellular protease $\beta$ was activated and extracellular protease synthesis and release commenced (Fig. I). The transfer procedure alone was without effect (Fig. 2).

Addition of protein (Table I) to the treatment media stimulated neither yield of extracellular protease (Fig. 2) nor accumulation of proteases within the mycelium. Instead there was a small, late reduction of yield (Fig. 2) as would be expected if the products of protein hydrolysis were leading to renewed repression.

Similar results, indicating the absence of induction, were obtained when mycelium was transferred to carbon-free medium with or without added protein. The subordinate role of induction was previously indicated by the fact that colonies growing on medium containing protein and Io mM-ammonium ion did not release protease (Cohen, 1972).

Derepression by carbon- or sulphur-starvation. Activation of protease $\beta$ together with synthesis and secretion of extracellular protease also followed transfer of mycelium from minimal medium to carbon-free or sulphur-free media (Fig. 3). Release of protease by carbon-starved mycelium was not stimulated in the presence of $1 \%(\mathrm{w} / \mathrm{v})$ protein, or thermolysin (Calbiochem Ltd, Io Wyndham Place, London W.I, $3 \times$ cryst. Lot 201095), $4 \mu \mathrm{g} / \mathrm{ml}$, or protein + thermolysin (see Drucker, 1973; and personal communication).

Derepression by phosphorus starvation. During growth on limiting phosphate and following transfer to phosphorus-free medium, conversion of the intracellular protease from precursor to active form occurred but the extracellular proteases were neither synthesized nor released (Fig. 3 and $4(a)-(f)$.

Inhibition of protease derepression by cycloheximide. After transfer to carbon-free or to nitrogen-free medium containing cycloheximide $(5 \mu \mathrm{g} / \mathrm{ml}$ and $100 \mu \mathrm{g} / \mathrm{ml})$, none of the changes associated with derepression was observed.

Regulation of proteases in xprDI. This mutant was isolated by selection for colonies able to release protease in the presence of ammonium (Cohen, 1972). When $x p r D_{I}$ was grown in glucose-ammonium liquid minimal medium, the intracellular protease $\beta$ occurred in its precursor forms but the extracellular proteases were synthesized and released with a specific activity higher than that of fully nitrogen-starved biAI. When $x p r D I$ was transferred to 


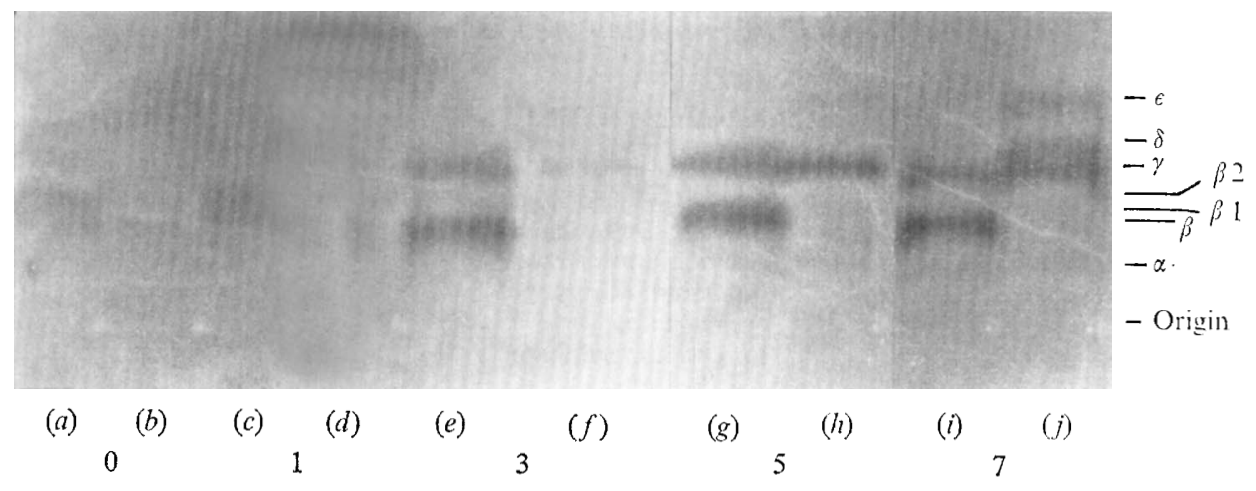

Time after transfer to $\mathrm{N}$-free medium biAl (h)

Fig. I. Electrophoresis, showing derepression of neutral and alkaline proteases of Aspergillus nidulans after transfer to nitrogen-free minimal medium; $(a),(c),(e),(g)$ and $(i)$ are mycelial extracts, and $(b)$, $(d),(f),(h)$ and $(j)$ are the corresponding culture filtrates.

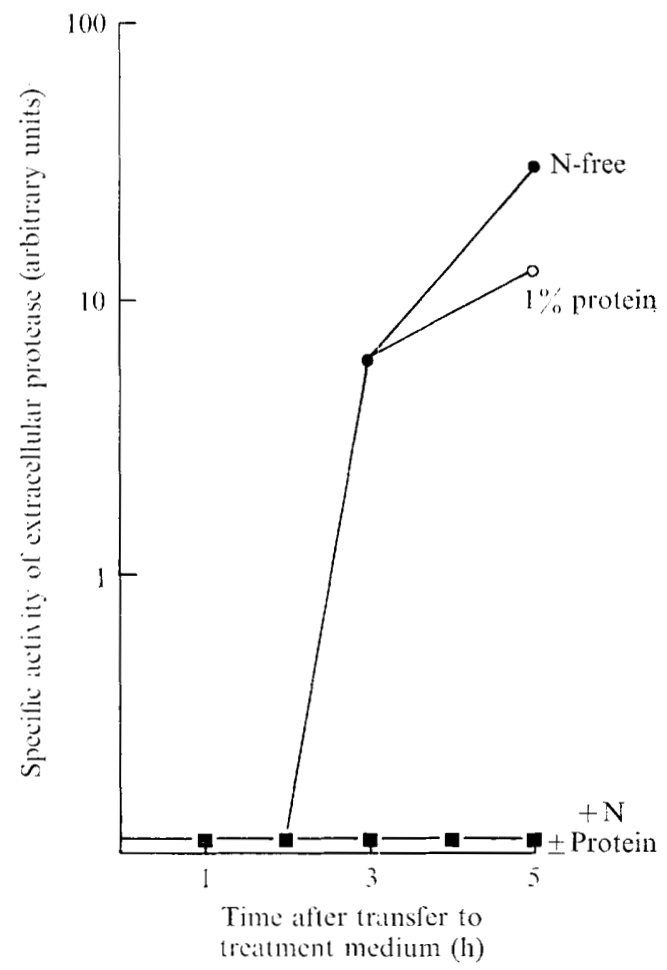

Fig. 2

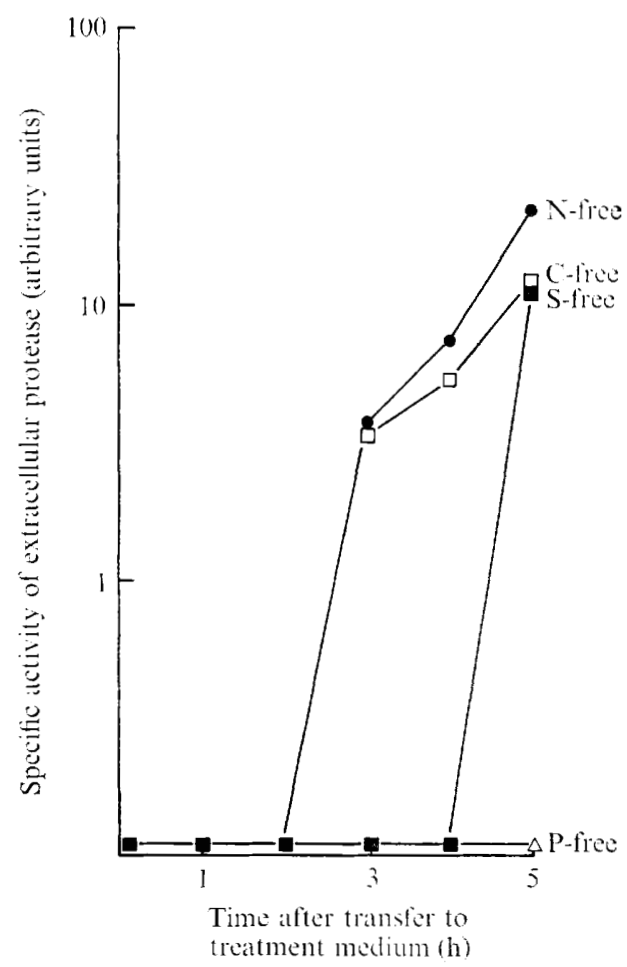

Fig. 3

Fig. 2. Specific activity (arbitrary units $/ \mathrm{mg}$ dry wt mycelium $/ \mathrm{ml}$ culture filtrate) of extracellular protease of Aspergillus nidulans after transfer to treatment media. Each point is the mean of three independent replicate experiments. $\mathbf{\square}$, Transfer from minimal medium to fresh minimal medium or to minimal medium containing $\mathrm{I} \%$ protein. O, Transfer to nitrogen-free minimal medium. 0 , Transfer to minimal medium containing $1 \%$ protein as sole nitrogen source.

Fig. 3. Specific activity (arbitrary units $/ \mathrm{mg}$ dry wt mycelium $/ \mathrm{ml}$ culture filtrate) of extracellular protease of Aspergillus nidulans after transfer to treatment media. Each point is the mean of two independent replicate experiments. - Transfer from minimal medium to nitrogen-free medium. $\square$, Transfer from minimal medium to carbon-free medium. $\mathbf{\square}$, Transfer from minimal medium to sulphur-free medium $\triangle$, Transfer from limiting-phosphorus minimal medium to phosphorus-free medium. 


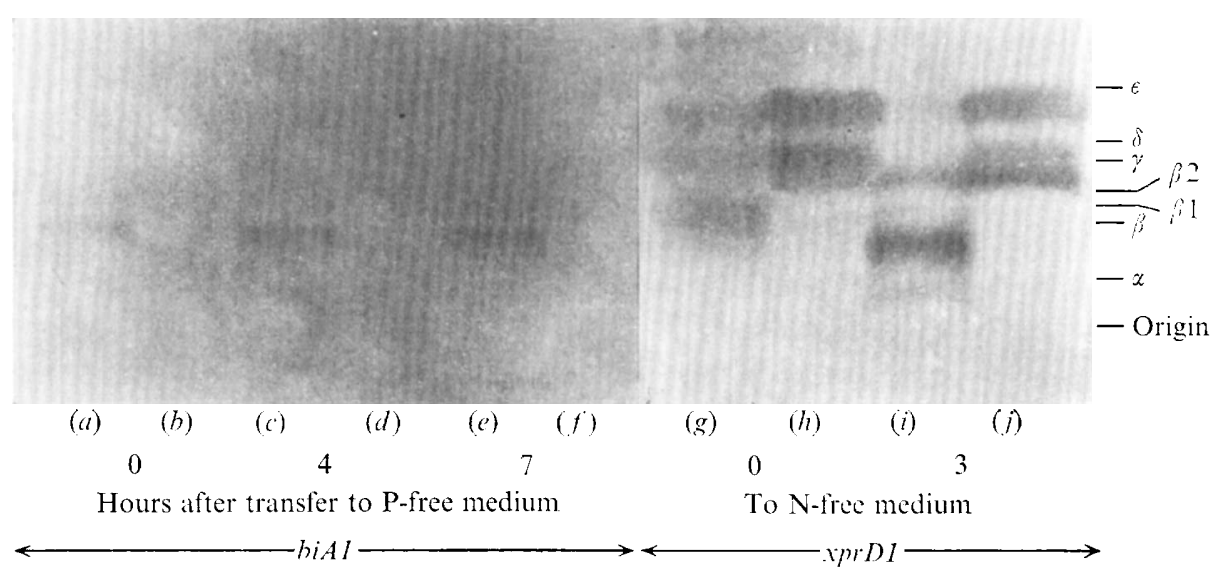

Fig. 4. Electrophoresis of neutral and alkaline proteases of Aspergillus nidulans. $(a)$ - $(f)$, Transfer of biAI from limiting-phosphorus minimal medium to phosphorus-free medium. $(g)$ - $(j)$, Transfer of $x p r D I$ from minimal medium to nitrogen-free medium. $(a),(c),(e),(g),(i)$, Mycelial extracts. $(b),(d),(f),(h),(j)$, The corresponding culture filtrates.

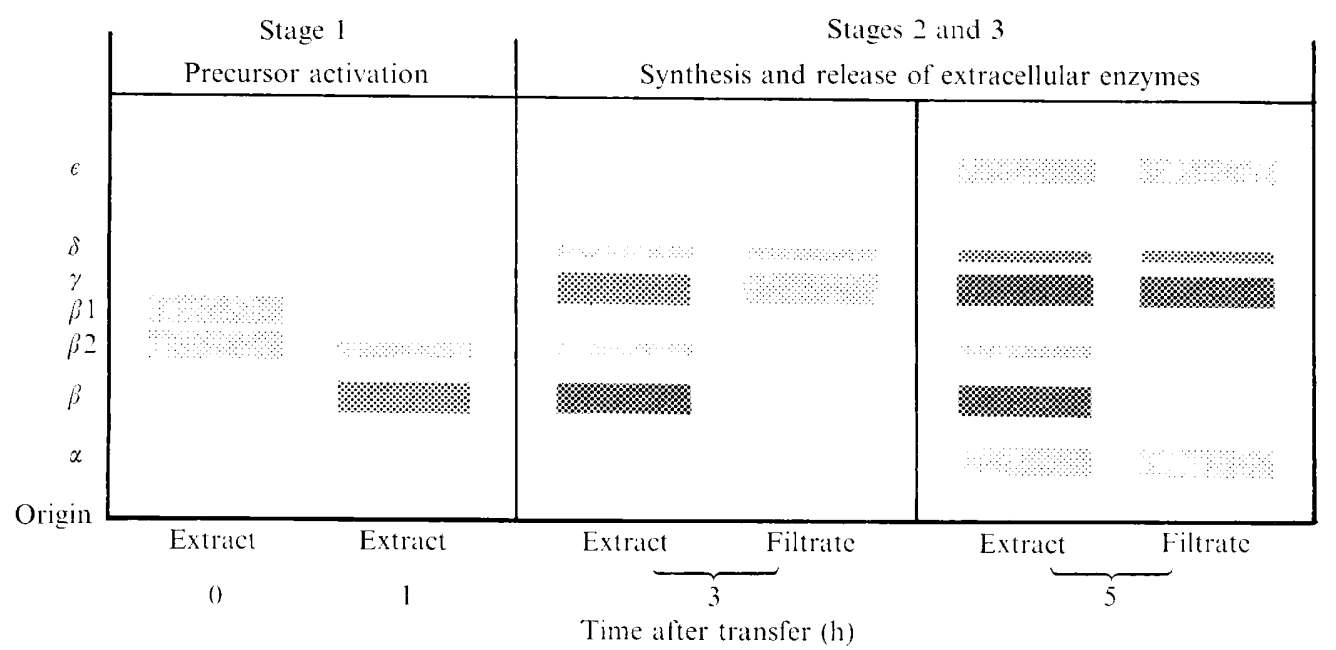

Fig. 5. Electrophoretic analysis of derepression of neutral and alkaline proteases of Aspergillus nidulans. This diagram summarizes the results of many experiments.

nitrogen- or carbon-free media, activation of the $\beta$ precursors occurred as expected (Fig. $4 \mathrm{~g}$, $j$ ). The defect in this mutant is thus confined to failure to repress synthesis and release of extracellular proteases, indicating the lack of relation between stage $I$ and stages 2 and 3 of the derepression process (Fig. 5 and below).

General features of derepression. The changes undergone by the intracellular and extracellular proteases of $b i A I$ in response to the transfer of mycelium from minimal medium to medium deficient in carbon or nitrogen or sulphur are summarized in Fig. 5 and appear to fall into three stages: stage I, activation of the precursors of intracellular protease $\beta$; stage 2 , synthesis of extracellular proteases; stage 3 , secretion of extracellular proteases (in order of quantity: $\delta-\gamma, \epsilon, \alpha)$. Stage I alone occurs in response to phosphorus-starvation and remains repressible in the mutant $x p r D_{I}$. It is therefore unconnected with the regulation of 


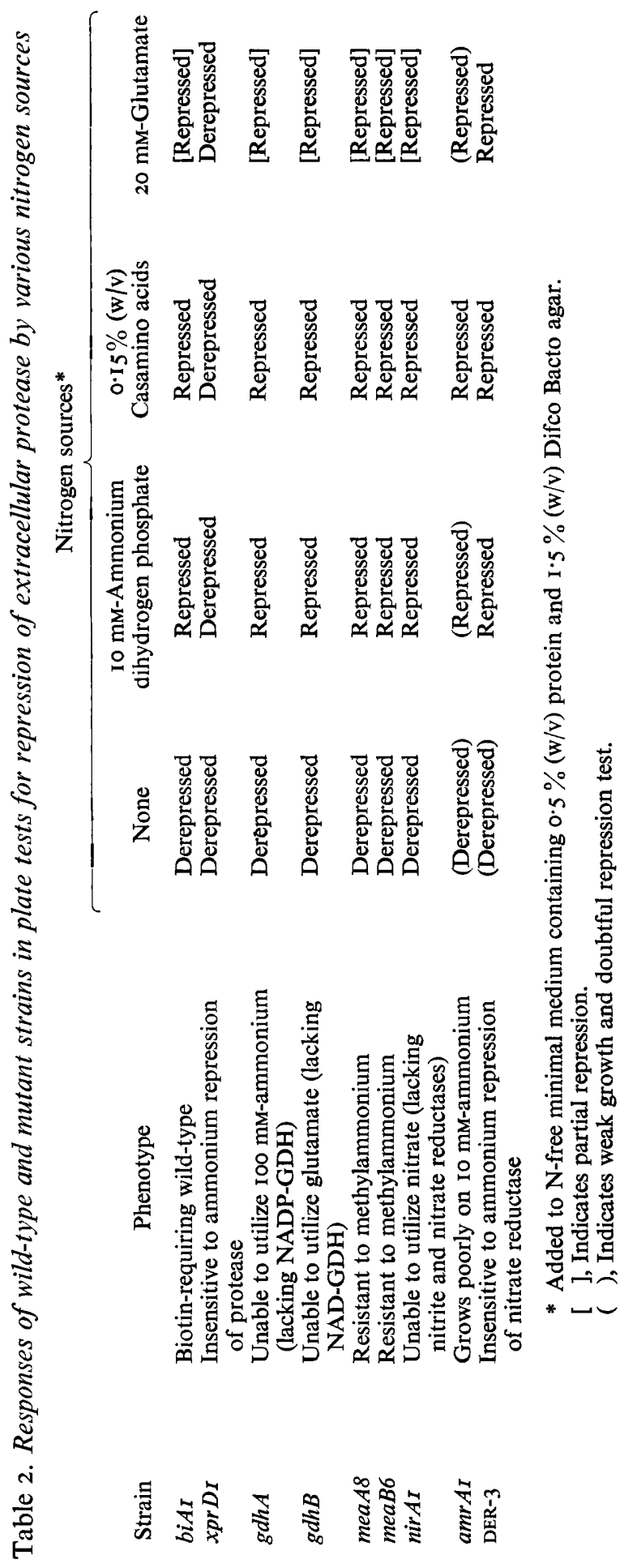


Protease regulation in Aspergillus nidulans

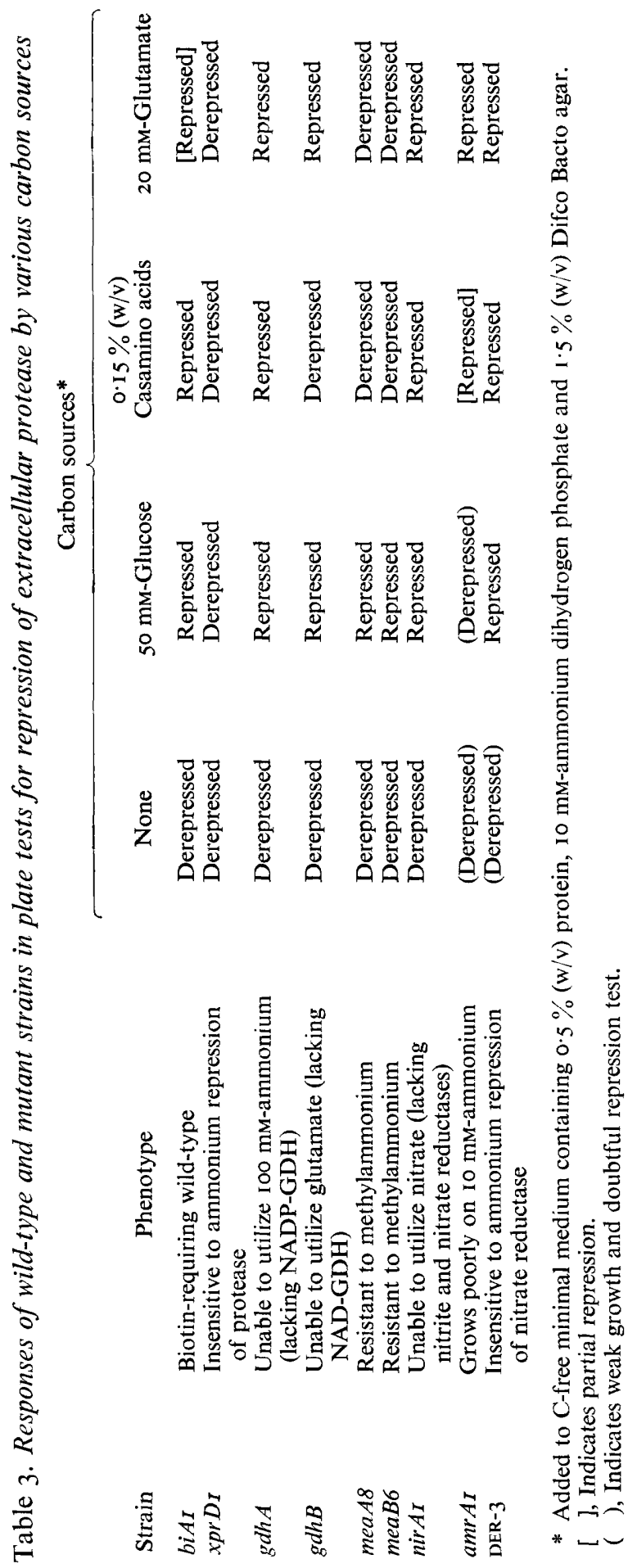


extracellular protease synthesis and can be regarded as a response to general starvation or unbalanced growth; it may be concerned with mobilization of intracellular proteins.

Stages 2 and 3 are closely associated, but are uncoupled in certain circumstances (unpublished observations). They occur in response to carbon or nitrogen or sulphur starvation and are not repressible in $x p r D I$.

Regulation of proteases in other mutants with abnormalities of ammonium repression. The results of plate tests for repression of protease by ammonium, glucose, Casamino acids and glutamate, acting, where appropriate, either as sole nitrogen or sole carbon source, are presented in Tables 2 and 3. The mutants tested include all classes known to have defective ammonium repression.

A plate test for protease repression by sulphur-containing metabolites was not available.

\section{DISCUSSION}

Two distinct (but connected) systems of ammonium regulation have been recognized in Aspergillus nidulans: the uptake of ammonium (measured by ${ }^{14} \mathrm{C}$-methylammonium transport) is regulated by a system responding to the internal ammonium concentration, whilst the synthesis of a variety of nitrogen catabolic enzymes and the activities of the urea and glutamate uptake systems are regulated by a system responding to the external ammonium concentration. Both of these systems of control become insensitive to ammonium repression in $g d h A$ mutants, and a hypothesis for the molecular mechanisms of these phenomena, in which NADP-GDH plays a dual role as enzyme and regulator, has been put forward (Pateman et al. 1973). A similar situation has been described in Saccharomyces cerevisiae by Grenson \& Hou (I972) and Dubois, Grenson \& Wiame (1973).

The characteristics of extracellular and intracellular protease regulation described here indicate that two new, independent regulatory systems, each involving repression, act on the proteases of Aspergillus nidulans. Regulation of extracellular protease synthesis and release appears to be a directly adaptive mechanism: when any one of carbon, nitrogen or sulphur is limiting growth, protease synthesis is released from repression and continues until the deficient metabolite is again available. For nitrogen, as ammonium, the threshold external concentration is as low as 2 to $5 \mathrm{~mm}$ (Cohen, 1972), whereas the internal ammonium concentration is at a very low level when external ammonium is at or below Io mM (J.A. Pateman, personal communication), indicating that the controlling effect of ammonium on protease is exerted externally or in an internal compartment too small to be detected by ammonium pool measurements. Further evidence for the importance of the external ammonium concentration in protease regulation is that when Io mM-urea is sole nitrogen source the internal ammonium concentration is high, yet protease is not fully repressed (Pateman et al. 1973; Cohen, 1972). No data indicate whether internal or external carbon and sulphur metabolite concentrations are important.

Regulation of activation of the precursors of the intracellular protease $\beta$ is through carbon, nitrogen, sulphur and phosphorus repression, activation following deprivation for any one of these elements. For nitrogen repression, the same arguments suggest that it is the extracellular concentration that is important; again no data indicate whether internal or external carbon, sulphur or phosphorus concentrations are important.

The independence of the protease regulatory systems from those previously described is demonstrated by the fact that in $g d h A$ and five other strains with defects of ammonium regulation, ammonium repression of protease is normal. Although the properties of $x p r D$ appear to offer evidence connecting the extracellular protease regulatory system with that 
controlling glutamate and urea uptake and the catabolic enzymes, this possibility requires further testing in the light of the finding that a Neurospora mutant with enhanced extracellular enzyme synthesis has a cell-wall defect (Gratzner, 1972) and the suggestion that xprD may have a cell-membrane defect (Pateman et al. 1973).

The results relating to carbon metabolite repression of protease are difficult to assess and it is not yet clear whether the abnormalities of carbon repression in several mutants, especially $g d h B$, meaA and meaB, are significant in the context of regulatory mechanisms. These results are, however, important because the insensitivity of $x p r D$ to carbon and sulphur repression, coupled with the effectiveness of carbon- and sulphur-starvation in derepressing protease synthesis in $b i A I$, point to the existence of a close connexion between nitrogen, carbon and sulphur repression and suggest that no study of the one can be complete without parallel study of the others. The existence of sulphur repression of proteases in Aspergillus niger was originally reported by Tomonaga, Ohama \& Yanagita (1964) and by Tomonaga (I966).

The regulation of proteases in Aspergillus nidulans by repression without induction may be contrasted with the results of Drucker (1972; 1973; and personal communication) who finds that in Neurospora crassa protease release requires an inductive interaction between exogenous protein, homologous or heterologous protease and intact mycelium as well as carbon starvation.

I am grateful to Miss Moira Hogg for excellent technical assistance.

\section{REFERENCES}

ARst, H. N. \& CoVE, D. J. (1969). Methylammonium resistance in Aspergillus nidulans. Journal of Bacteriology $98,1284-1293$.

Arst, H. N. \& PAGe, M. M. (1973). Mutants of Aspergillus nidulans altered in the transport of methylammonium and ammonium. Molecular and General Genetics I21, 239-245.

Cohen, B. L. (1972). Ammonium repression of extracellular protease in Aspergillus nidulans. Journal of General Microbiology 7r, 293-299.

COHEN, B. L. (1973a). Growth of Aspergillus nidulans in a thin liquid layer. Journal of General Microbiology $76,277-283$.

CoHEN, B. L. (I973 b). The neutral and alkaline proteases of Aspergillus nidulans. Journal of General Microbiology $77,521-528$.

DRUCKER, H. (1972). Regulation of extracellular proteases in Neurospora crassa: induction and repression of enzyme synthesis. Journal of Bacteriology II0, I04I-IO49.

DrUCKER, H. (1973). Regulation of extracellular proteases in Neurospora crassa: The role of Neurospora proteases in induction. Journal of Bacteriology (in the Press).

Dubois, E., Grenson, E. \& Wiame, J. M. (1973). Release of the 'ammonia effect' on three catabolic enzymes by NADP-specific glutamate dehydrogenaseless mutations in Saccharomyces cerevisiae. Biochemical and Biophysical Research Communications 50, 967-972.

GratZNER, H. G. (1972). Cell wall alteration associated with the hyperproduction of extracellular enzymes in Neurospora crassa. Journal of Bacteriology III, 443-446.

Grenson, M. \& Hou, C. (1972). Ammonia inhibition of the general amino acid permease and its suppression in NADP-specific glutamate dehydrogenaseless mutants of Saccharomyces cerevisiae. Biochemical and Biophysical Research Communications 48, 749-756.

Kinghorn, J. R. \& PATEMAN, J. A. (I973). NAD and NADP-glutamate dehydrogenase activity and ammonium regulation in Aspergillus nidulans. Journal of General Microbiology 78, 39-46.

Pateman, J. A. \& Cove, D. J. (1967). Regulation of nitrate reductase in Aspergillus nidulans. Nature, London 2I5, I234-I 237.

Pateman, J. A., Kinghorn, J. R., Dunn, E. \& Forbes, E. (1973). Ammonium regulation in Aspergillus nidulans. Journal of Bacteriology (in the Press). 
Pontecorvo, G., Roper, J. A., Hemmons, L. M., MacDonald, K. D. \& Bufton, A. W. J. (I953). The genetics of Aspergillus nidulans. Advances in Genetics 5, I4I-238.

TomonaGA, G. (I966). Preferential synthesis of extracellular protease by Aspergillus niger in sulphur deficiency. Journal of General and Applied Microbiology 12, 267-276.

Tomonaga, G., OHama, H. \& YanaGita, T. (1964). Effect of sulphur compounds on the protease formation by Aspergillus niger. Journal of General and Applied Microbiology ro, 373-386. 